\title{
LINE INTEGRAL APPROXIMATION OF DOUBLE INTEGRALS
}

\author{
H. D. BRUNK AND G. M. EWING
}

1. Introduction. It has been shown by Wilkins [2] and Grosswald [1] that the double integral of a continuous function $F(x, y)$ over a circular region centered at the origin is the limit of a related integral along the spiral $\theta=\alpha r$ as $\alpha \rightarrow \infty$. This result is generalized in several directions in the present paper.

The authors were led to this study by way of certain special cases arising in connection with the design of an analog computer at Sandia Corporation. This device among other things approximates the probability mass

$$
\frac{1}{2 \pi} \iint \exp \left(-r^{2} / 2\right) r d r d \theta
$$

for a wide variety of regions $R$, by scanning $R$ along arcs of a related spiral.

2. A general limit theorem. Let $R$ be a bounded, measurable subset of the plane. Let $f(r, \theta), g(r, \theta)$, and $h(r)$ be functions with the following properties:

(2.1) $f(r, \theta)$ is continuous on the closure $\bar{R}$ of $R$, and periodic in $\theta$ of period $2 \pi$.

(2.2) $h(r)$ is positive, almost everywhere on $[0, a]$, and is summable on $[0, a]$, where $a$ is the radius of a circle, centered at the origin, containing $\bar{R}$ in its interior.

It is convenient to the exposition to set $f(r, \theta) \equiv 0,(r, \theta) \notin \bar{R}$, and $h(r) \equiv 1, r>a$.

(2.3) $g(r, \theta) \equiv h(r) f(r, \theta)$, and hence is of period $2 \pi$ in $\theta$. It follows that $|g|$ is dominated on $R$ by a function summable over $R$ and hence that $g$ is summable over $R$.

We consider the integral

$$
I=\frac{1}{2 \pi} \iint g(r, \theta) d r d \theta,
$$

subject to conditions (2.1) to (2.3).

For $\alpha>0$, let $\Gamma_{\alpha}$ denote the spiral

Presented to the Society, April 26, 1952, under the title The approximation of double integrals by means of line integrals; received by the editors April 21, 1952 and, in revised form, June 23, 1952. 


$$
\theta=\alpha \int_{0}^{r} h(u) d u,
$$

and define a sequence $\left\{r_{i}\right\}$ by the equation

$$
2 i \pi=\alpha \int_{0}^{r_{i}} h(u) d u, \quad i=0,1,2, \cdots
$$

The set of points $(r, \theta)$ satisfying inequalities $r_{i} \leqq r \leqq r_{i+1}$ will be denoted by $S_{i}$. Let $C_{i}$ denote any of the class of arcs in $S_{i}$ on each of which $r$ is nondecreasing in $\theta(0 \leqq \theta \leqq 2 \pi)$. Let

(2.7) $\quad Q_{i}^{+} \equiv \sup _{C_{i}} \int_{C_{i} \cap R} f(r, \theta) d \theta, \quad$ and $\quad Q_{i}^{-} \equiv \inf _{C_{i}} \int_{C_{i} \cap R} f(r, \theta) d \theta$.

For each $r>0$, let $E_{r}$ denote the set of $\theta$-values corresponding to all points $(r, \theta)$ in $R$. As a consequence of (2.3) and Fubini's theorem, we have

$$
I=\frac{1}{2 \pi} \int_{0}^{\infty} h(r) d r \int_{E_{r}} f(r, \theta) d \theta .
$$

Our approximating line integral is

$$
I^{*} \equiv \frac{1}{\alpha} \int_{\Gamma_{\alpha} \cap_{R}} f(r, \theta) d \theta=\int_{\Gamma_{\alpha} \cap_{R}} g(r, \theta) d r .
$$

Let

$$
I_{i} \equiv \frac{1}{2 \pi} \int_{r_{i}}^{r_{i}+1} h(r) d r \int_{E_{r}} f(r, \theta) d \theta
$$

and

$$
I_{i}^{*} \equiv \frac{1}{\alpha} \int_{\Gamma_{\alpha} \cap R \cap s_{i}} f(r, \theta) d \theta=\int_{\Gamma_{\alpha} \cap \cap_{R} \cap s_{i}} g(r, \theta) d r
$$

Clearly

$$
I=\sum_{0}^{\infty} I_{i}=\sum_{0}^{m-1} I_{i} \quad \text { and } \quad I^{*}=\sum_{0}^{\infty} I_{i}^{*}=\sum_{0}^{m-1} I_{i}^{*}
$$

where $m=m(\alpha)$ is the largest integer such that $r_{m} \leqq a$. We consider only values of $\alpha$ such that $R$ is in the circle of radius $r_{m}$ centered at the origin. It follows from definitions (2.7) and (2.11) that

$$
Q_{i}^{-} \leqq \alpha I_{i}^{*} \leqq Q_{i}^{+} \text {. }
$$


We see from (2.6) and (2.10) that $I_{i}$ satisfies these same inequalities and hence that

$$
\left|I_{i}-I_{i}^{*}\right| \leqq \frac{1}{\alpha}\left(Q_{i}^{+}-Q_{i}^{-}\right) .
$$

It then follows from (2.12) that

$$
\left|I-I^{*}\right| \leqq \frac{1}{\alpha} \sum\left(Q_{i}^{+}-Q_{i}^{-}\right) .
$$

Given $\epsilon>0$ and $i$, a positive integer or zero, definitions (2.7) imply the existence of functions $\lambda_{i}(\theta)$ and $\mu_{i}(\theta)$, each bounded by $r_{i}$ and $r_{i+1}$ and monotone nondecreasing on $[0,2 \pi]$, such that

$$
\left|Q_{i}^{+}-\int_{0}^{2 \pi} f\left[\lambda_{i}(\theta), \theta\right] \phi_{i}(\theta) d \theta\right|<\epsilon,
$$

and

$$
\left|Q_{\bar{i}}-\int_{0}^{2 \pi} f\left[\mu_{i}(\theta), \theta\right] \psi_{i}(\theta) d \theta\right|<\epsilon
$$

in which

$$
\begin{aligned}
\phi_{i}(\theta) & =1 \text { if }\left[\lambda_{i}(\theta), \theta\right] \in R \\
& =0 \text { otherwise, }
\end{aligned}
$$

and similarly for $\psi_{i}(\theta)$. It follows that

$$
Q_{i}^{+}-Q_{i}^{-}<2 \epsilon+\int_{0}^{2 \pi} \Delta(\theta) d \theta
$$

where $\Delta \equiv\left|f\left[\lambda_{i}(\theta), \theta\right] \phi_{i}(\theta)-f\left[\mu_{i}(\theta), \theta\right] \psi_{i}(\theta)\right|$.

For fixed $i$, let $s_{i}$ denote the set of $\theta$-values for which either $(r, \theta)$ $\in R$ for each $r$ on $\left[r_{i}, r_{i+1}\right]$, or $(r, \theta) \notin R$ for each $r$ on $\left[r_{i}, r_{i+1}\right]$. Thus, for $\theta \in s_{i}$, either both $\left(\lambda_{i}(\theta), \theta\right)$ and $\left(\mu_{i}(\theta), \theta\right)$ are in $R$, or neither is. Let $s_{i}^{\prime}$ be the complementary set, $[0,2 \pi]-s_{i}$. It follows from (2.1) and from (2.2) and (2.6), which imply that $\left(r_{i+1}-r_{i}\right) \rightarrow 0$ uniformly with respect to $i$ as $\alpha \rightarrow \infty$, that there exists a positive number $A$ such that

$$
\Delta<\epsilon, \quad \text { for } \theta \in s_{i} \text {, if } \alpha>A \text {, independently of } i \text {. }
$$

Let $M$ be a bound on $|f(r, \theta)|$ for $(r, \theta) \in R$. Then

$$
\Delta \leqq 2 M \quad \text { for arbitrary } \theta, i .
$$


It follows from (2.6), (2.13), (2.17), (2.18), and (2.19) that, for $\alpha>A$,

$$
\left|I_{i}-I_{i}^{*}\right|<\left[\frac{\epsilon}{\pi}+\frac{1}{2 \pi} \int_{s_{i}} \Delta d \theta+\frac{1}{2 \pi} \int_{s_{i}^{\prime}} \Delta d \theta\right] \cdot \int_{r_{i}}^{r_{i}+1} h(r) d r,
$$

and hence that

$$
\left|I_{i}-I_{i}^{*}\right|<\epsilon\left(\frac{1}{\pi}+1\right) \int_{r_{i}}^{r_{i+1}} h(r) d r+\frac{2 M}{\alpha} m\left(s_{i}^{\prime}\right),
$$

where $m\left(s_{i}^{\prime}\right)$ denotes the measure of the set $s_{i}^{\prime}$. The bounded set $R$ is inside the circle of radius $a$ with center at the origin. It follows from (2.12) and (2.20) that

$$
\left|I-I^{*}\right| \leqq 2 \epsilon \int_{0}^{a} h(r) d r+\frac{2 M}{\alpha} \sum m\left(s_{i}^{\prime}\right) .
$$

Corresponding to each partition $P: 0=r_{0}<r_{1}<r_{2}<\cdots<r_{n}=a$ of $[0, a]$ is a sum $\sum m\left(s_{i}^{\prime}\right)$. We now impose upon $R$ the condition that

$$
\sup _{P} \sum m\left(s_{i}^{\prime}\right)<\infty \text {. }
$$

The following theorem is then a consequence of (2.21).

THEOREM 2.1. If $R$ is a bounded measurable set with property (2.22), then $I=\lim I^{*}$ as $\alpha \rightarrow \infty$.

It is clear from the definition of the sets $s_{i}$ that if no point of the boundary of $R$ is in $S_{i}$ and on a given ray from the origin, then the $\theta$-value corresponding to that ray belongs to $s_{i}$. Thus $s_{i}^{\prime}$ is contained in the set of $\theta$-values subtended at the origin by the part of the boundary of $R$ in $S_{i}$. It is then clear that if $R$ is a region on the boundary of which $\theta$ is of bounded variation, then $R$ satisfies condition (2.22). In particular, if the boundary of the region $R$ is rectifiable, and if the origin is not on the boundary (from which it follows that $\theta$ is of bounded variation), then $R$ has property (2.22).

If the origin $O$ is on the rectifiable boundary, $C$, of $R$, and if an arc of $C$ containing $O$ in its interior is a straight line, then also it is clear that $\theta$ is of bounded variation. Suppose then that $C$ is rectifiable, that $O$ is on $C$, and that no arc of $C$ containing $O$ is rectilinear. To each positive number $\epsilon$ corresponds a positive number $\delta$ such that all points at distance less than $\delta$ along $C$ from $O$ lie in a circle of center $O$ and radius $\epsilon$. Choose two such points, $P_{1}$ and $P_{2}$, on opposite sides of $O$ and not on the same straight line with $O$; form the boundary of a region $R^{\prime}$ bounded by the rectifiable curve $C^{\prime}$ which is $C$, with the arc $P_{1} P_{2}$ replaced by the straight line $P_{1} P_{2}$. Since $O$ is not on the 
boundary of $R^{\prime}$, Theorem 2.1 applies to $R^{\prime}$. From (2.1)-(2.3), (2.8), and (2.9), it is clear that the integrals $I$ and $I^{*}$ corresponding to regions contained in a circle of radius $\epsilon$ are arbitrarily small for sufficiently small $\epsilon$. It follows that $\lim _{\alpha \rightarrow \infty} I^{*}=I$ for $R$, since $R-R^{\prime}$ lies in the circle with center $O$ and radius $\epsilon$. We have then the following corollary of Theorem 2.1:

If $R$ is bounded by a finite set of rectifiable simple closed curves, then $I=\lim I^{*}$ as $\alpha \rightarrow \infty$, irrespective of the choice of origin.

3. Integrands in which variables are separable. We now consider the special case in which $f$ is independent of $r$. We retain hypotheses (2.1) to (2.3) and assume further that $f(\theta) \geqq 0$.

A region bounded by two rays from the origin and an arc with polar equation $r=F(\theta), F(\theta)$ continuous and single-valued, will be called a wedge. If $F(\theta)$ and $G(\theta)$ are both continuous and single-valued with $0 \leqq F(\theta) \leqq G(\theta)$, the region bounded by two rays from the origin and by arcs with respective equations $r=F(\theta)$ and $r=G(\theta)$ will be called a truncated wedge. If $F(\theta) \equiv 0$, the truncated wedge becomes a wedge. The two bounding segments of rays are sides of a wedge or truncated wedge, and the angle between 0 and $2 \pi$ subtended by the boundary is the angle of the wedge or truncated wedge. The point of intersection of the sides is the vertex.

Lemma 3.1. If $R$ is a wedge of angle $\phi$ with sides $\theta=\theta_{1}$, and $\theta=\theta_{1}+\phi$, and if $k$ is any positive integer or zero, then

$$
\sum_{0}^{k}\left(Q_{i}^{+}-Q_{i}^{-}\right) \leqq \int_{\theta_{1}}^{\theta_{1}+\phi} f(\theta) d \theta .
$$

Proof. It follows from definition (2.7), the definition of the sets $E_{r}$, and the single-valuedness of $F(\theta)$, that

$$
Q_{\bar{i}}^{-}=\int_{E_{i+1}} f(\theta) d \theta, \text { and } Q_{i}^{+}=\int_{E_{i}} f(\theta) d \theta .
$$

Here and elsewhere in this section $E_{i}$ means $E_{r}, r=r_{i}$.

The set $E_{r}$ for a wedge is monotone nonincreasing in $r$ since $F(\theta)$ is single-valued. Thus $E_{r}$ has a limit as $r \rightarrow 0$. This limit defines $E_{0}$ appearing in the second integral (3.1) when $i=0$. We find by addition that

$$
\sum_{0}^{k}\left(Q_{i}^{+}-Q_{i}^{-}\right)=Q_{0}^{+}-Q_{\bar{k}+1}=\int_{E_{0}} f(\theta) d \theta-\int_{E_{k+1}} f(\theta) d \theta .
$$

The first term on the right does not exceed the right member of the 
relation we wish to prove. Hence the lemma is true as stated.

LEMMA 3.2. If $R$ is a truncated wedge of angle $\phi$, with sides $\theta=\theta_{1}$, and $\theta=\theta_{1}+\phi$, if there exists a number $r^{*}$ such that $F(\theta) \leqq r^{*} \leqq G(\theta)$, and if $k$ is any positive integer or zero, then

$$
\sum_{0}^{k}\left(Q_{i}^{+}-Q_{i}^{-}\right) \leqq 2 \int_{0_{1}}^{\theta_{1}+\phi} f(\theta) d \theta .
$$

Proof. Let $j$ denote the largest non-negative integer such that $r_{j} \leqq r^{*}$. The set $E_{r}$ is monotone nondecreasing in $r$ for $0 \leqq r \leqq r^{*}$ but monotone nonincreasing for $r>r^{*}$. It follows that

$$
\begin{array}{rlrl}
Q_{i}^{-} & =\int_{E_{i}} f(\theta) d \theta, & & i<j, \\
& =\min \left\{\int_{E_{i}} f(\theta) d \theta, \int_{E_{i+1}} f(\theta) d \theta\right\}, & i=j, \\
& =\int_{E_{i+1}} f(\theta) d \theta, & i>j,
\end{array}
$$

while

$$
\begin{aligned}
Q_{i}^{+} & =\int_{E_{i+1}} f(\theta) d \theta, & i<j, \\
& =\int_{E_{r^{*}}} f(\theta) d \theta, & i=j, \\
& =\int_{E_{i}} f(\theta) d \theta, & i>j .
\end{aligned}
$$

If $Q_{\bar{j}}$ has the first alternative value, we find that

$$
\begin{aligned}
\sum_{0}^{k}\left(Q_{i}^{+}-Q_{i}^{-}\right) & =\int_{E_{r^{*}}}+\int_{E_{j+1}}-\int_{E_{0}}-\int_{E_{k+1}} f(\theta) d \theta, & k>j, \\
& =\int_{E_{k+1}}-\int_{E_{0}} f(\theta) d \theta, & k \leqq j .
\end{aligned}
$$

None of the integrals $\int_{E_{r^{*}},} \int_{E_{j+1}}, \int_{E_{k+1}} f(\theta) d \theta$ exceeds

$$
\int_{0_{1}}^{\theta_{1}+\phi} f(\theta) d \theta \text {. }
$$

The lemma follows in this case from (3.2), and the assumption that 
$f \geqq 0$. If $Q_{\bar{j}}^{-}$has the second value, the only change is to replace subscript $j+1$ by $j$ in the second integral of (3.2).

LEMMA 3.3. The interior of every truncated wedge $W$ is the union of a countable set of nonoverlapping truncated wedges corresponding to each of which is a number $r^{*}$ with the property stated in the preceding lemma. Moreover, the sum of the angles of these truncated wedges does not exceed the angle of $W$.

Proof. If $F(\theta) \equiv G(\theta)$, or if the angle of $W$ is zero, there are no interior points, and the lemma is vacuously true. Suppose next that there is an interior point. Through each interior point $(r, \theta)$ of $W$ pass circular arcs of radius $r$, all points of which are interior to $W$. Rays from the origin through the ends of such an arc bound, with $r=F(\theta)$ and $r=G(\theta)$ (the bounding arcs of $W$ ), a truncated wedge $w$ contained in $W$. Let $I$ denote the open $\theta$-interval subtended by the interior of $w$.

Consider the class $\{I\}$ of all such $I$. By the Lindelöf Covering Theorem, there exists a countable subset of $\{I\}$, which we denote by

$$
I_{1}, I_{2}, I_{3}, \cdots \text {, }
$$

which covers the open set of $\theta$-values corresponding to interior points of $W$. By subtracting the union of preceding intervals from each interval $I_{n}$, we obtain a countable set of nonoverlapping intervals. The union of the corresponding truncated wedges is the interior of $W$. It is clear that the sum of their angles is not greater than the angle of $W$ (it may be less; e.g., if $F \equiv G$ on an interval).

One can show by examples that "countable" cannot be replaced by "finite" in this lemma.

A decomposition of a region $R$ into wedges, or into truncated wedges, will be called simple if no two have corresponding $\theta$-intervals with common interior points. It follows that the sum of the angles of the wedges (truncated wedges) of such a decomposition is not greater than $2 \pi$. From Lemmas 3.1 to 3.3, relation (2.14), and the observation that both $I$ and $I^{*}$ are (finitely) additive with respect to disjoint regions $R$, we have the following theorems:

TheOREM 3.1. If $R$ admits a simple decomposition into a countable set of wedges with vertices at the origin, then

$$
\left|I-I^{*}\right| \leqq \frac{1}{\alpha} \int_{0}^{2 \pi} f(\theta) d \theta .
$$

THEOREM 3.2. If $R$ admits a simple decomposition into a countable set of truncated wedges with vertices at the origin, then 


$$
\left|I-I^{*}\right| \leqq \frac{2}{\alpha} \int_{0}^{2 \pi} f(\theta) d \theta .
$$

We have remarked that a wedge is a truncated wedge. Hence Theorem 3.2 applies to mixed decompositions in which some of the truncated wedges reduce to wedges.

We have been considering functions $f$ which are not only independent of $r$, but are also non-negative. Dropping this restriction, we have the theorem:

THEOREM 3.3. If $R$ admits a simple decomposition into a countable set of wedges with vertices at the origin, on each of which $f(\theta)$ is of constant sign, then

$$
\left|I-I^{*}\right| \leqq \frac{1}{\alpha} \int_{0}^{2 \pi}|f(\theta)| d \theta .
$$

The corresponding generalization of Theorem 3.2 is also immediate.

Theorem 3.3 is applicable, for example, to a double integral of a power function $x^{m} y^{n}$, when it is transformed into polar coordinates.

It follows from any of the preceding theorems that $I=\lim I^{*}$ as $\alpha \rightarrow \infty$, as has been shown under more general hypotheses in Theorem 2.1.

THEOREM 3.4. Let $R$ be $a$ circular disc of radius $b<a$. Let the parameter $\alpha$ of (2.6) be such that the point $\left(b, \theta_{1}\right)$ is on the spiral $\Gamma_{\alpha}$. A necessary and sufficient condition in order that $I=I^{*}$, without passage to the limit, is that

$$
\frac{1}{\theta_{1}} \int_{0}^{\theta_{1}} f(\theta) d \theta=\frac{1}{2 \pi} \int_{0}^{2 \pi} f(\theta) d \theta .
$$

In particular, $I=I^{*}$ if the spiral makes an integral number of turns in $R$.

Proof. Let $k$ be the largest integer such that $r_{k}<b$. Then from (2.10) and (2.11), we have

$$
I_{i}=I_{i}^{*}=\frac{1}{2 \pi} \int_{0}^{2 \pi} f(\theta) d \theta \int_{r_{i}}^{r_{i+1}} h(r) d r, \text { for } 0 \leqq i<k,
$$

since, by (2.6),

$$
\frac{1}{2 \pi} \int_{r_{i}}^{r_{i+1}} h(r) d r=\frac{1}{\alpha}
$$


Also

$$
I_{k}=\frac{1}{2 \pi} \int_{0}^{2 \pi} f(\theta) d \theta \int_{r_{k}}^{b} h(r) d r
$$

while

$$
I_{k}^{*}=\frac{1}{2 \pi} \int_{0}^{\theta_{1}} f(\theta) d \theta \int_{r_{k}}^{r_{k+1}} h(r) d r
$$

By (2.5),

$$
\int_{r_{k}}^{b} h(r) d r=\frac{\theta_{1}}{\alpha}
$$

while

$$
\frac{1}{2 \pi} \int_{r_{k}}^{r_{k+1}} h(r) d r=\frac{1}{\alpha} .
$$

Hence

$$
I-I^{*}=I_{k}-I_{k}^{*}=\frac{\theta_{1}}{2 \pi \alpha} \int_{0}^{2 \pi} f(\theta) d \theta-\frac{1}{\alpha} \int_{0}^{\theta_{1}} f(\theta) d \theta .
$$

The conclusion of the theorem is immediate.

\section{BIBLIOGRAPHY}

1. E. Grosswald, On the integration scheme of Marechal, Proceedings of the American Mathematical Society vol. 2 (1951) pp. 706-709.

2. J. E. Wilkins, Jr., An integration scheme of Marechal, Bull. Amer. Math. Soc. vol. 55 (1949) pp. 191-192.

Sandia Corporation and

UNIVERSITY OF MISSOURI 